\title{
TRANSTHORACIC ECHOCARDIOGRAPHY AND DEPENDENCE OF ELECTROCARDIOGRAPHIC INDICATORS ON PHYSICAL ACTIVITY TYPE IN LITHUANIAN ATHLETES
}

\author{
Julija Borkytė, Renata Žumbakytė-Šermukšnienė \\ Lithuanian University of Health Sciences, Kaunas, Lithuania
}

\begin{abstract}
Background. Since athletes have a higher risk to experience cardiovascular system complications, this gives us an important reason for further investigation. Early detection of pathological hypertrophy of the left ventricle may exclude athletes from sports activities and prevent complications and possible death.

Methods. In our study, 75 patients underwent transthoracic echocardiography (TTE). The values of left ventricle were measured: left ventricle end-diastolic diameter (LVEDD), interventricular septum thickness in diastole (IVSTd), LV posterior wall thickness in diastole (LVPWTd), LV mass (LVM), LV mass index (LVMI), LV end-diastolic diameter index (LVEDDI). Standard 12-lead ECG was recorded at $25 \mathrm{~mm} / \mathrm{s}$ and with standard calibration of $10 \mathrm{~mm} /$ $\mathrm{mV}$ from a patient in a supine position.

Results. When we compared results of LVH according to ECG and TTE, ECG showed higher count of athletes with marginal changes and less LVH compared to TTE in female endurance and male athletes. Moreover, moderate positive correlation between LV mass and R wave size (V5) $(r=.617)$ was found in female endurance athletes. Furthermore, high positive correlation between LVEDD and QRS interval length $(r=.911)$ was found in female strength athletes. Also, moderate negative correlation $(r=-.603)$ between heart electrical axis and $\mathrm{R}$ wave size (V5) was found in male endurance athletes.

Conclusions. Knowledge of the ECG changes associated with the type and intensity of exercise, race, age and gender can lower the traditionally high number of false positives, thus reducing unnecessary investigations. In our study, ECG showed higher count of athletes with marginal changes and less LVH compared to TTE in women endurance, men strength and endurance groups.
\end{abstract}

Keywords: heart ventricles, electrocardiography, hypertrophy.

\section{INTRODUCTION}

$\mathrm{S}$ udden death is a tragic event in any occasion. Sudden death during sports is an extremely rare event, but the impact is greater, because athletes are regarded as the healthiest group in society. ECG can reveal changes associated with cardiac disease. Puig, Freitas, Costa, and de Freitas (1994) have concluded that ECG changes in athletes usually reflect structural adaptation to regular physical training. On the other hand, abnormalities on a young athlete's ECG can also be an expression of an underlying disease that may carry a risk of sudden cardiac death (SCD) during sports. Lack of knowledge of the 'normal' ECG changes in athletes may lead to false-positive results and often limits participation in sports to athletes whose ECG patterns are within the normal pattern.

Current European Society of Cardiology (ESC) guidelines (Uberoi et al., 2011) include an ECG, besides physical examination, as a prescreening test for athletes. The American Heart Association (AHA) and the ESC have both published recommendations (Corrado et al., 2010) 
for the interpretation of the ECG in athletes. These guidelines presents a summary of the most frequent ECG changes considered 'normal' in athletes and creates discussion regarding the advantages and disadvantages of ECG screening in this population.

Athlete's heart is currently defined (Leite, Freitas, Campelo, \& Maciel, 2016) as a nonpathological condition as a result of structural and morphological adaptation to intensive exercise. The cardiovascular adaptation to exercise differs according to physical activity type: endurance (dynamic/aerobic) and strength training (isometric/ anaerobic). Some sports, such as cycling and rowing, combine both types of cardiovascular changes. Venckunas, Stasiulis, and Raugale (2006) suggest that endurance sports lead to volume overload and increased diameter of the heart chambers, while strength sports can cause overload by pressure and are related to the thickening of the left ventricle wall. Early detection of pathological hypertrophy of the left ventricle may exclude athletes from sports activities and prevent complications and death. At present echocardiography can analyse (D'Andrea et al., 2006) the structural and functional changes in the myocardium of athlete's hearth and distinguish the physiological and pathological hypertrophy.

While studies are so controversial and similar studies have not been carried out in Lithuania, it is relevant to find more information about transthoracic echocardiography and electrocardiographic indicators' dependence on physical activity type in Lithuanian athletes.

\section{METHODS}

The study was carried out at the Kaunas Sports Medicine Centre within 2 years. The Bioethics Centre's Approval was received at LSMU (No. BEC - MF - 474) in order to publish the article. Patients were selected based on the following criteria: age (from 18 to 40 years), athletes, who were engaged in strength or endurance sports at least $4 \mathrm{~h} /$ week for 1 year. Patients arrived at a periodic medical examination at Kaunas County Medical Centre where they underwent twodimensional transthoracic echocardiography (TTE). Their systolic and diastolic blood pressure, height, weight and history of exercise intensity were noted. Hasdemir et al. (2011) suggested that arterial blood pressure should be measured in the subject's sitting position by auscultation of Korotkoff sounds (first and fifth Korotkoff tone phase) with a stethoscope and using a cuff before performing (TTE). Also, none of our subjects had physical activity on the day of the study. Standard 12-lead electrocardiogram (ECG) was recorded from a patient in a supine position on the same day of examination (before TTE).

As Yim (2013) suggested, we chose to perform a two-dimensional transthoracic echocardiography because it is non-invasive investigation and does not involve radiation. We used ultrasound system CX50 with transducer - S5-1 in this investigation. A two dimensional Doppler technique was used as well. We used these dimensions to measure the left ventricular: left ventricle end-diastolic diameter in diastole (LVEDD, mm), interventricular septum thickness in diastole (IVSTd, mm), LV posterior wall thickness in diastole (LVPWTd, mm). Lang et al. (2015) recommend to measure these indicators at end of the diastole (before the mitral valve closing or in the heart cycle with the largest diameter or volume of the ventricle). Due to the statistically significant difference between the subjects' height and weight in the groups of athletes, it was decided to evaluate the relative indices with the area of the body surfaces. Relative body surface area was calculated according to the formula:

$$
\begin{aligned}
& \mathrm{LVM}=0.8 \times(1.04((\text { LVEDD }+ \text { LVPWTd }+ \\
& \left.\left.\mathrm{IVST}^{3}-\mathrm{LVEDD}^{3}\right)\right)+0.6 \mathrm{~g},
\end{aligned}
$$

where LVM - left ventricular myocardial mass; LVEDD - left ventricle end-diastolic diameter; IVSTd - interventricular septum thickness in diastole; LVPWTd - LV posterior wall thickness in diastole, based on the latest recommendations of the American Society of Echocardiography (2015), and the European Cardiovascular Association (Lang et al., 2015). According to these parameters, the following values were calculated: LV mass (LVM, g), LVMI (LV mass index, $\mathrm{g} / \mathrm{m}^{2}$ ), LV enddiastolic diameter index (LVEDDI, $\mathrm{mm} / \mathrm{m}^{2}$ ). The left ventricular myocardial mass index (LVMI) was calculated dividing the mass of the left ventricular by the body surface area. The left ventricular diastolic diameter index (LVEDDI) was calculated dividing the diastolic size of the left ventricle by the body surface area.

Statistical analysis was performed using SPSS version 16.0 (IBM, Armonk, NY, USA) software package. Qualitative variables were described based on their frequency rate (\%). The quantitative values are given as the arithmetic mean \pm standard deviation $(\mathrm{x} \pm S D)$. Qualitative variables' homogeneous distribution was evaluated by chi-square $\left(\chi^{2}\right)$ test $\left(\chi^{2}\right.$ or Fisher's exact test - in 
case of small expected values). The normality of distribution of quantitative variables was tested by Kolmogorov-Smirnov test. For comparison of quantitative variables Mann-Whitney-Wilcoxon (for non-normal distributions), Student's $T$-test (for normally distributed values) were used. The correlation coefficient $(r)$ of Spearman, Pearson, and Cramér's V was calculated for the relation between variables. The relation when the correlation coefficient $r<.3$ was considered as weak, when $.3 \leq$ $r \leq .7$ was considered as moderate and when $r>$ .7 was considered as strong. Differences at $p<.05$ were considered as statistically significant.

\section{RESULTS}

This study involved 75 participants (age $23.39 \pm 5.86)$ in total. Individuals were categorized by gender and physical activity type (strength and endurance athletes): 20 women were endurance sports representatives, while strength sports group involved 5 subjects; 25 men were endurance sports volunteers, when 25 subjects were categorized as strength sports athletes. General characteristics of the athletes are presented in Table 1.

When we compared results of LVH according to ECG and TTE, ECG showed higher count of athletes with marginal changes and less LVH compared to TTE (Figures 1, 2) in women endurance, men strength and endurance groups.

In our study, moderate positive correlation between LVEDDI and QRS interval length ( $r=$ .486 ) and between IVSTd and R wave size (V5) $(r=.453)$ was found in female endurance athletes. Moreover, moderate positive correlation between LV mass and R wave size (V5) $(r=.617)$ was

Table 1. Athletes' characteristics by gender

\begin{tabular}{|c|c|c|c|c|}
\hline Subjects' groups & $\begin{array}{l}\text { Women endurance } \\
\text { athletes } \\
(n=20)\end{array}$ & $\begin{array}{c}\text { Women strength } \\
\text { athletes } \\
(n=5)\end{array}$ & $\begin{array}{c}\text { Men endurance } \\
\text { athletes } \\
(n=25)\end{array}$ & $\begin{array}{l}\text { Men strength } \\
\text { athletes } \\
(n=25)\end{array}$ \\
\hline Age (years) & $24.14 \pm 6.16$ & $20.38 \pm 2.45$ & $22.35 \pm 5.35$ & $24.44 \pm 6.46$ \\
\hline Height (cm) & $175.30 \pm 7.94$ & $162.80 \pm 7.5$ & $186.08 \pm 8.77$ & $183.44 \pm 8.69$ \\
\hline Weight (kg) & $69.80 \pm 11.52$ & $60.00 \pm 8.31$ & $81.04 \pm 9.87$ & $85.16 \pm 16.73$ \\
\hline Body surface area $\left(\mathrm{m}^{2}\right)$ & $1.83 \pm 0.18$ & $1.63 \pm 0.13$ & $2.03 \pm 0.15$ & $2.03 \pm 0.22$ \\
\hline Years of training (years) & $13.25 \pm 7.09$ & $6.60 \pm 3.29$ & $12.20 \pm 6.86$ & $10.84 \pm 5.76$ \\
\hline Hours of training (hours/week) & $9.40 \pm 4.28$ & $6.80 \pm 2.08$ & $11.20 \pm 6.06$ & $8.42 \pm 2.68$ \\
\hline Systolic blood pressure (mmHg) & $115.20 \pm 9.81$ & $114.40 \pm 11.61$ & $123.76 \pm 12.43$ & $126.24 \pm 9.55$ \\
\hline Diastolic blood pressure (mmHg) & $70.20 \pm 10.80$ & $64.80 \pm 8.67$ & $73.92 \pm 13.39$ & $76.80 \pm 6.89$ \\
\hline Resting heart rate $(\mathrm{t} / \mathrm{min})$ & $67.50 \pm 10.64$ & $70.80 \pm 12.30$ & $70.52 \pm 9.62$ & $66.96 \pm 7.88$ \\
\hline
\end{tabular}

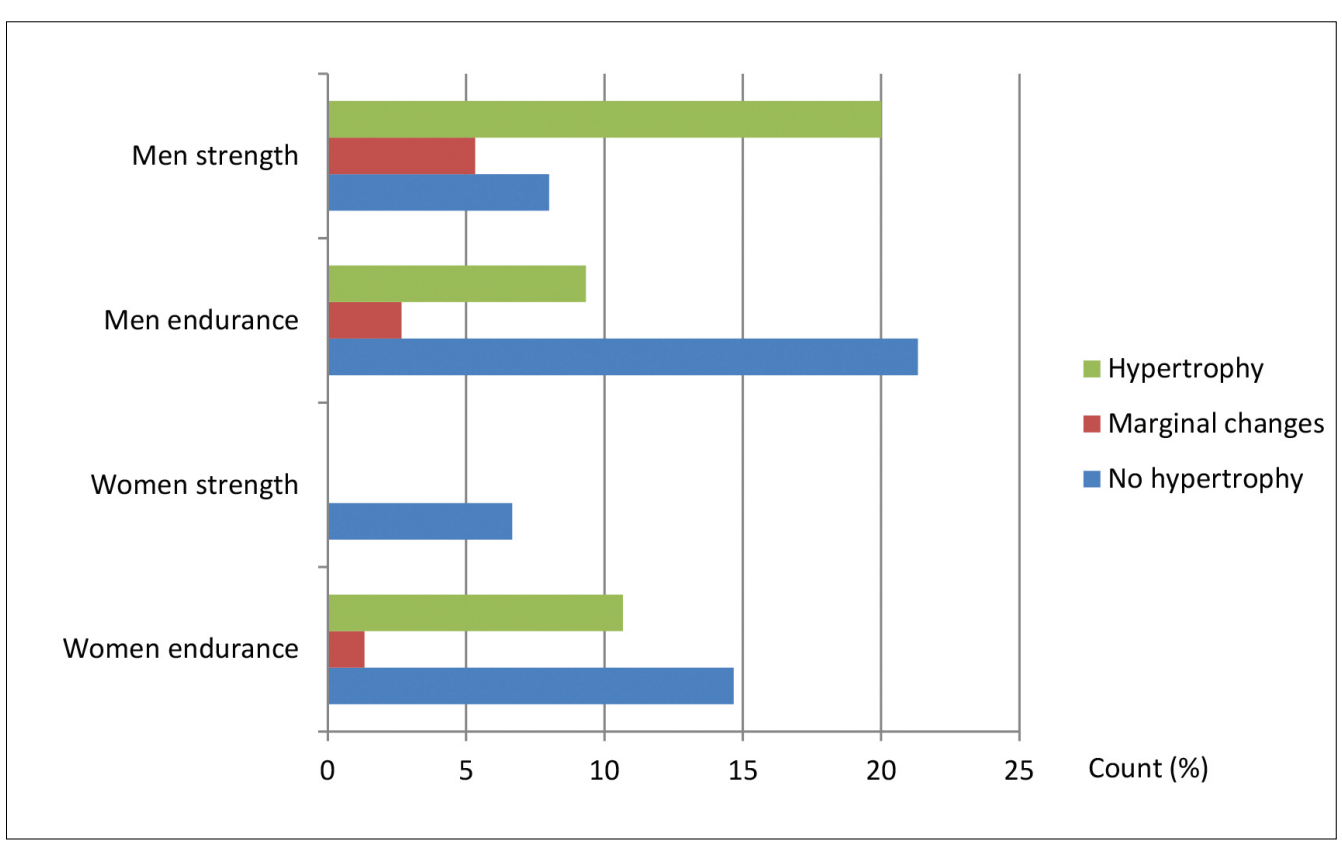

Figure 1. LVH according to transthoracic echocardiography 
Figure 2. LVH according to ECG

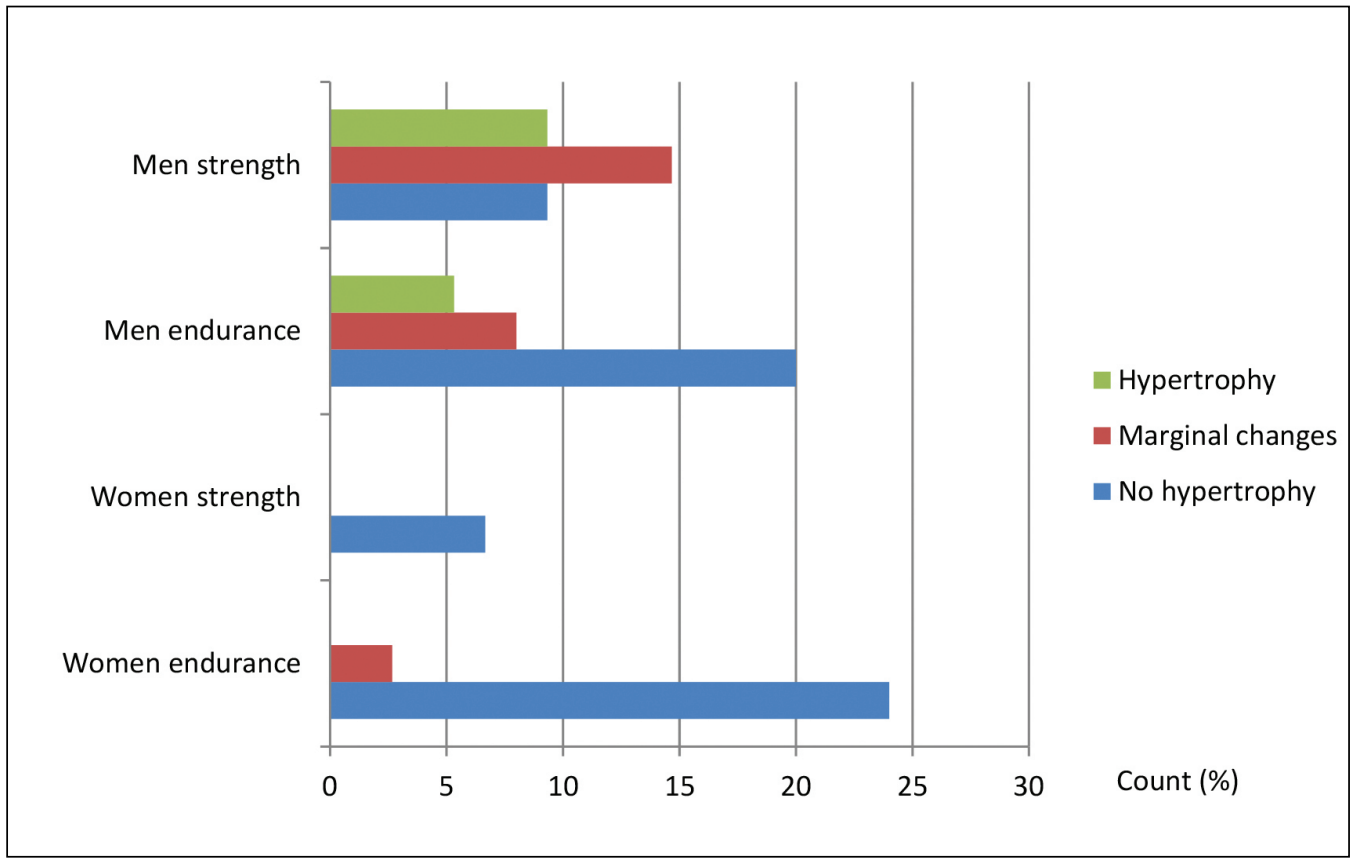

Table 2. LVH ECG an TTE parameters according to gender and physical activity type

\begin{tabular}{|l|c|c|c|c|}
\hline \multicolumn{1}{|c|}{ Subjects' groups } & $\begin{array}{c}\text { Women endurance } \\
\text { athletes }\end{array}$ & $\begin{array}{c}\text { Women strength } \\
\text { athletes }\end{array}$ & $\begin{array}{c}\text { Men endurance } \\
\text { athletes }\end{array}$ & $\begin{array}{c}\text { Men strength } \\
\text { athletes }\end{array}$ \\
\hline LVEDD $(\mathrm{mm})$ & $46.57 \pm 3.56$ & $41.30 \pm 3.35$ & $51.02 \pm 3.19$ & $50.92 \pm 4.83$ \\
\hline LVEDDI $\left(\mathrm{mm} / \mathrm{m}^{2}\right)$ & $25.59 \pm 2.55$ & $25.50 \pm 2.97$ & $25.24 \pm 1.95$ & $25.09 \pm 2.31$ \\
\hline LVM $(\mathrm{g})$ & $149.11 \pm 29.56$ & $96.22 \pm 21.25$ & $197.25 \pm 28.20$ & $215.42 \pm 49.06$ \\
\hline LVMI $\left(\mathrm{g} / \mathrm{m}^{2}\right)$ & $81.07 \pm 12.70$ & $58.94 \pm 11.64$ & $99.06 \pm 18.88$ & $105.08 \pm 18.40$ \\
\hline IVSTd $(\mathrm{mm})$ & $9.24 \pm 1.14$ & $7.72 \pm 1.07$ & $10.28 \pm 0.86$ & $10.95 \pm 1.25$ \\
\hline LVPWTd $(\mathrm{mm})$ & $9.45 \pm 1.10$ & $7.68 \pm 0.84$ & $10.29 \pm 0.82$ & $10.98 \pm 1.10$ \\
\hline QRS $(\mathrm{ms})$ & $89.45 \pm 10.78$ & $81.60 \pm 7.93$ & $95.68 \pm 10.69$ & $91.68 \pm 9.29$ \\
\hline Sokolov-Lyon criteria $(\mathrm{mm})$ & $24.60 \pm 5.30$ & $24.60 \pm 6.47$ & $29.80 \pm 10.79$ & $32.40 \pm 10.29$ \\
\hline Axis $\left({ }^{\circ}\right)$ & $60.80 \pm 35.51$ & $68.00 \pm 16.60$ & $75.00 \pm 28.51$ & $62.08 \pm 19.16$ \\
\hline
\end{tabular}

found in female endurance athletes. Also, high positive correlation between LVEDD and QRS interval length $(r=.911)$ was found in female strength athletes. Furthermore, moderate negative correlation between heart electrical axis and $\mathrm{R}$ wave size (V5) was found ( $r=-.603)$ was found in male endurance athletes. Also, moderate negative correlation between S wave size (V6) $(r=-.469)$ and LVEDDI was found in male strength athletes. General TTE and ECG characteristics of athletes are presented in Table 2 .

\section{DISCUSSION}

Dumanoir, Haykowsky, Syrotuik, Taylor, and Bell (2007); Gates, Tanakaa, Graves and Seals (2003) found that the impact of physical training on cardiac structure and function depended on the type, intensity and duration of training, as well as previous physical fitness, genetics and gender. Investigation on the physiological limits of left ventricular hypertrophy in elite junior athletes (Sharma et al., 2002) demonstrated that the left ventricular posterior wall thickness in the group of athletes was significantly increased compared to the untrained subjects. Pluim, Zwinderman, Van der Laarse, Van der Around and Wall (2000) studied the hearts of 1451 athletes and concluded that the absolute average thickness of the posterior wall of the left ventricle and the interventricular septum in the control group was significantly lower than that in the strength, endurance, or concurrent training groups. Also, Mahdiabadi, Gaeini, Kazemi and Mahdiabadi (2013) found that the thickness of 
the left ventricle, the interventricular septum and the posterior wall was the greatest in the strength group. According to the latest recommendations (2015), American Society for Echocardiography and the European Cardiovascular Association (Lang et al., 2015) provided standards based on body surface area. The present study highlights the poor accuracy of the five tested ECG criteria in correctly identifying LVH based on the results of the gold standard echocardiogram method in a population of athletes. The Perugia, Cornell and Romhilt- Estes ECG criteria presented high negative predictive values, which could be helpful for excluding the presence of LVH in athletes. (Samesima et al. (2017) found that the Perugia and Cornell criteria were more effective for excluding LVH in athletes involved in sport modalities with a predominance of the dynamic component.

The greatest disadvantage of pre-participation screening of young athletes' ECGs for abnormalities that may disclose a cardiac disease is the high number of false positives. Corrado et al. (2010) found that unawareness of the changes regarded as normal on young athletes' ECGs could lead to these being classified as abnormal in up to $50 \%$ of athletes. Knowledge of the ECG changes associated with the type and intensity of exercise, race, age and gender can lower the high number of false positives, and reduce unnecessary investigations.

\section{CONCLUSIONS}

A large number of young athletes demonstrate ECG changes due to physical adaptation and only a very small number actually have changes that are considered abnormal and need further investigation. Knowledge of the ECG changes associated with the type and intensity of exercise, race, age and gender can lower the traditionally high number of false positives, thus reducing unnecessary investigations. Our study found that ECG showed higher count of athletes with marginal changes and less LVH compared to TTE in women endurance, men strength and endurance groups

Acknowledgements. None of the authors declare competing financial interests. This research did not receive any specific grant from funding agencies in the public, commercial, or not-for-profit sectors.

Conflict of interest declaration. The authors have no conflict of interest to declare.

\section{REFERENCES}

Corrado, D., Pelliccia, A., Heidbuchel, H., Sharma, S., Link, M., Basso, C., ... McKenna, W. J. (2010). Recommendations for interpretation of 12 - lead electrocardiogram in the athlete. European Heart Journal, 31(2), 243-259. doi: 10.1093/eurheartj/ehp473

D'Andrea, A., D'Andrea, L., Caso, P., Scherillo, M., Zeppilli, P., \& Calabrò, R. (2006). The usefulness of Doppler myocardial imaging in the study of athlete's heart in the differential diagnosis between physiological and pathological ventricular hypertrophy. Echocardiography, 23(2), 149-157. doi: 10.1111/j.15408175.2006.00186.x

Dumanoir, G. R., Haykowsky, M. J., Syrotuik, D. G., Taylor, D. A., \& Bell, G. J. (2007). The effect of high intensity rowing and combined strength and endurance training on left ventricular systolic function and morphology. International Journal of Sports Medicine, 28(6), 488-494. doi: 10.1055/s-2006-955897

Gates, P. E., Tanakaa, H., Graves, J., \& Seals, D. R. (2003). Left ventricular structure and diastolic function with human ageing. Relation to habitual exercise and arterial stiffness. European Heart Journal, 24(24), 2213-2220.

Hasdemir, H., Yildiz, M., Metin, G., Kasap, H., Yildiz, B. S., Yaylak, B., \& Özyurt, A. (2011). Aortic properties and atrial electrophysiology in the young and old football players. Revista da Associação Médica Brasileira, 57(3), 280-285.

Lang, R. M., Badano, L. P., Mor-Avi, V., Afilalo, J., Armstrong, A., Ernande, L., ... Voigt, J. U. (2015). Recommendations for cardiac chamber quantification by echocardiography in adults: An update from the American society of echocardiography and the European association of cardiovascular imaging. Journal of the American Society of Echocardiography, 28(1), 1-39. e14. doi: 10.1016/j.echo.2014.10.003

Leite, S. M., Freitas, J., Campelo, M., \& Maciel, M. J. (2016). Electrocardiographic evaluation in athletes: 'Normal' changes in the athlete's heart and benefits and disadvantages of screening. Revista Portuguesa de Cardiologia, 35(3), 169-177. doi: 10.1016/j. repc.2015.09.024

Mahdiabadi, J., Gaeini, A. A., Kazemi, T., \& Mahdiabadi, M. A. (2013). The effect of aerobic continuous and interval training on left ventricular structure and function in male non-athletes. Biology of Sport, 30(3), 207-211. doi: 10.5604/20831862.1059302 Pelliccia, A., Di Paolo, F. M., De Blasiis, E., Quattrini, F. M., Pisicchio, C., Guerra, E., ... Maron, B. J. (2010). Prevalence and clinical significance 
of aortic root dilatation in highly trained competitive athletes. Circulation, 122(7), 698-706. doi: 10.1161/ CIRCULATIONAHA.109.901074

Pluim, B. M., Zwinderman, A. H., Van der Laarse, L. A., Van der Around, V., \& Wall, E. E. (2000). The athletes heart a meta-analysis of cardiac structure and function. Circulation, 101(3), 336-344.

Puig, J., Freitas, J., Costa, O., \& de Freitas, A. F. (1994). Sudden death in athletes. Revista Portuguesa de Cardiologia, 13(1), 59-62.

Samesima, N., Azevedo, L. F., Echenique, L. S., Negrao, C. E., Pastore, C. A., \& Pastore, C. A. (2017). Comparison of electrocardiographic criteria for identifying left ventricular hypertrophy in athletes from different sports modalities. Clinics (Sao Paulo), 72(6), 343-350. doi: 10.6061/clinics/2017(06)03.

Sharma, S., Maron, B. J., Whyte, G., Firoozi, S., Elliott, P. M., \& McKenna, W. J. (2002). Physiologic limits of left ventricular hypertrophy in elite junior athletes: Relevance to differential diagnosis of athlete's heart and hypertrophic cardiomyopathy. Journal of the American College of Cardiology, 40(8), 1431-1436.

Uberoi, A., Stein, R., Perez, M. V., Freeman, J.,
Wheeler, M., Dewey, F., ... \& Froelicher, V. (2011). Interpretation of the electrocardiogram of young athletes. Circulation, 124(6), 746-757. doi: 10.1161/ CIRCULATIONAHA.110.013078

Venckunas, T., Stasiulis, A., \& Raugale, R. (2006). Concentric myocardial hypertrophy after one year of increased training volume in experienced distance runners. British Journal of Sports Medicine, 40(8), 706709. doi: 10.1136/bjsm.2006.027813

Verma, S., \& Siu, S. C. (2014). Aortic dilatation in patients with bicuspid aortic valve. The New England Journal of Medicine, 370(20), 1920-1929. doi: 10.1056/ NEJMra1207059

Zeppilli, P., Vannicelli, R., Santini, C., Dello Russo, A., Picani, C., Palmieri, V., ... Pietrangeli, L. (1995). Echocardiographic size of conductance vessels in athletes and sedentary people. International Journal of Sports Medicine, 16(1), 38-44. doi: 10.1055/s-2007972961

Yim, E. S. (2013). Aortic root disease in athletes: Aortic root dilatation, anomalous coronary artery, bicuspid aortic valve, and Marfan's syndrome. Sports Medicine, 43(8), 721-732. doi: 10.1007/s40279-013-0057-6 